\section{Simple and Rapid Determination of Organochlorine Pesticide Residues in Kampo Products by Gas Chromatography/Mass Spectrometry with Negative Chemical lonization}

\author{
Takaomi Tagami, ${ }^{*}$ Keiji Kajimura, \\ Katsuhiro Yamasaki, Yoshiyuki Sawabe, \\ Chie Nomura, Shuzo Taguchi, \\ and Hirotaka Obana
}

Osaka Prefectural Institute of Public Health, 1-3-69 Nakamichi, Higashinari-ku, Osaka 537-0025, Japan

(Received October 27, 2009; Accepted November 23, 2009; Published online November 27, 2009)

A simple, rapid and inexpensive sample preparation method using extraction by shaking and sulfuric acid treatment was developed for determining eight organochlorine pesticides in kampo products by gas chromatography/mass spectrometry with negative chemical ionization. The recoveries were between $71 \%$ and $117 \%$, and most relative standard deviations were less than $10 \%$. The proposed method was applied to twenty-three samples of kampo formulae, and no samples were contaminated by eight organochlorine pesticides over the quantification limit.

Key words — kampo product, pesticide residue, gas chromatography/mass spectrometry

\section{INTRODUCTION}

Kampo formulae are used for various illnesses, and these formulae should be confirmed to be safe because patients generally take kampo formulae directly over the long term. $\alpha$-benzene hexachloride (BHC), $\beta$-BHC, $\gamma$-BHC, $\delta$-BHC (BHCs), $p, p^{\prime}-$ dichloro diphenyl dichloroethylene (DDE), $o, p^{\prime}-$ dichloro diphenyl trichloroethane (DDT), $p, p^{\prime}$ dichloro diphenyl dichloroethane (DDD) and $p, p^{\prime}-$

${ }^{*}$ To whom correspondence should be addressed: Osaka Prefectural Institute of Public Health, 1-3-69 Nakamichi, Higashinari-ku, Osaka 537-0025, Japan. Tel.: +81-6-69721321; Fax: +81-6-6972-2393; E-mail: tagami@iph.pref.osaka. jp
DDT (DDTs) have been detected in crude drugs ${ }^{1-3)}$ that are the ingredients of kampo formulae, and there is concern that BHCs and DDTs will move from crude drugs to kampo products. The levels of BHCs and DDTs in kampo formulae are controlled to be lower than self-imposed residual pesticide limits set by the Japan Kampo Medicine Manufacturers Association. Maximum residue levels of organochlorine pesticides were set for kampo formulae containing ginseng, senna leaf, astragalus root, polygala root, glycyrrhiza, cinnamon bark, asiasarum root, cornus fruit, perilla herb, jujube, citrus unshiu peel, loquat leaf and/or moutan bark [BHCs: $0.2 \mathrm{ppm}$ (as total BHCs), DDTs: $0.2 \mathrm{ppm}$ (as total DDTs)]. However, there have been few surveys of BHCs and DDTs in kampo products distributed in Japan, and few analytical methods have been reported. ${ }^{1,4,5)}$ All methods are time-consuming and use gas chromatography (GC)/electron capture detection (ECD). It was reported previously that organochlorine pesticides, such as BHCs and DDTs in crude drugs, could be analyzed selectively by $\mathrm{GC} /$ mass spectrometry (MS) with negative chemical ionization (NCI). ${ }^{6}$ ) Furthermore, a simple and rapid determination of pyrethroid pesticides in kampo products by GC/MS with NCI was also reported. ${ }^{7)}$ In this study, a simple, rapid, and inexpensive sample preparation method to determine BHCs and DDTs in kampo products was investigated using GC/MS with NCI.

\section{MATERIALS AND METHODS}

Pesticide Standards — Pesticide standards were obtained from Wako Pure Chemical Industries (Osaka, Japan) and Riedel de Haën (Hannover, Germany). Each compound was dissolved in acetone to make $0.5 \mathrm{mg} / \mathrm{ml}$ standard stock solution. From these, spiking solutions were prepared at $5 \mu \mathrm{g} / \mathrm{ml}$.

Reagents - Acetone, hexane, and anhydrous sodium sulfate of pesticide analysis grade and sulfuric acid were purchased from Wako Pure Chemical Industries.

Kampo Products - Nine kampo formulae (kamishoyosan, hochuekkito, daikenchuto, saireito, shosaikoto, bakumondoto, goshajinkigan, rikkunshito and shoseiryuto) of the top 10 by amount of importation and production ${ }^{8)}$ were used in this study. Self-imposed residual pesticide limits are applied to these 9 kampo formulae. Three kampo 
products were obtained for each kampo formulae for which self-imposed residual pesticide limits were set. Saireito and daikenchuto consisted of 2 samples because these kampo formulae are distributed by only 2 companies. Goshajinkigan consisted of 1 sample because this kampo formula is distributed by only 1 company. Overall, 23 kampo products of 9 kampo formulae were obtained (year: 2009).

Sample Preparation - Kampo products were powdered, $5 \mathrm{~g}$ was weighed out, and spiking solution (each pesticide: $1 \mu \mathrm{g}$ ) was added. After $30 \mathrm{~min}$, a total of $10 \mathrm{ml}$ of acetone and $20 \mathrm{ml}$ of hexane were added. The mixture was shaken for $30 \mathrm{~min}$, centrifuged, and the supernatant was recovered. Water $(20 \mathrm{ml})$ was added to the supernatant. The mixture was shaken for $5 \mathrm{~min}$. A portion $(5 \mathrm{ml})$ of the upper layer was transferred to a glass-stoppered test tube, and $1 \mathrm{ml}$ of sulfuric acid was added and shaken carefully. A portion $(4 \mathrm{ml})$ of the upper layer was taken, transferred to a glass-stoppered test tube, and $2 \mathrm{ml}$ of water was added and shaken gently. A portion $(3 \mathrm{ml})$ of the upper layer was taken, transferred to a tube, and dried with $1 \mathrm{~g}$ of anhydrous sodium sulfate and the supernatant was used as the sample solution. NCI Mode GC/MS — A 5973MSD was connected to a GC6890 (Agilent, Wilmington, DE, U.S.A.). GC conditions: column, DB-1701 capillary column $30 \mathrm{~m} \times 0.25 \mathrm{~mm} \times 0.25 \mu \mathrm{m}(\mathrm{J} \& \mathrm{~W}$ Scientific, Folsam, CA, U.S.A.); helium carrier gas flow, $1 \mathrm{ml} / \mathrm{min}$; injection temperature, $200^{\circ} \mathrm{C}$; interface temperature, $270^{\circ} \mathrm{C}$; ion source temperature, $180^{\circ} \mathrm{C}$; ion mode, $\mathrm{NCI} /$ selected ion monitoring (SIM) mode; reaction gas, methane; oven temperature program, $50^{\circ} \mathrm{C}$ for $1 \mathrm{~min},+30^{\circ} \mathrm{C} / \mathrm{min}$ to $100^{\circ} \mathrm{C}$, and then $+20^{\circ} \mathrm{C} / \mathrm{min}$ to $270^{\circ} \mathrm{C}$ and held for $10 \mathrm{~min}$; injection mode, splitless; injection volume, $2 \mu \mathrm{l}$.

\section{RESULTS AND DISCUSSION}

The monitoring ions selected for SIM detection are shown in Table 1. Values of $71(\mathrm{~m} / z$ : BHCs $)$ and $35(\mathrm{~m} / z$ :DDTs $)$ were used for quantification. The analytical method was investigated using kamishoyosan. Sample preparation method was based on the previous report. ${ }^{7)}$ When the recovery test was conducted according to the previous report, ${ }^{7)}$ a matrix enhancement effect was observed. So the sample preparation method was slightly changed and sulfuric treatment of the extraction liquid was supplemented. It was reported that sulfuric treatment is effective for purifying BHCs and DDTs. $\left.{ }^{9}{ }^{9}, 10\right)$ One of the previous reported methods for the analysis of BHCs and DDTs in kampo products also used sulfuric acid treatment in combination with column clean-up and liquid-liquid partition. ${ }^{4)}$ Sulfuric acid treatment purified extraction liquid by degradation of organic substances. BHCs and DDTs were not decomposed by sulfuric acid treatment because they are acid-stable. Sulfuric acid treatment was suitable for this study because it was simple and inexpensive. Intra-day recovery study (BHCs and DDTs: 3 spike concentrations)

Table 1. Monitoring Ions Selected for SIM

\begin{tabular}{lcc}
\hline \hline Compound & Monitoring ion $(m / z)$ \\
\hline$\alpha$-BHC & 71 & 35 \\
$\gamma$-BHC & 71 & 35 \\
$\beta$-BHC & 71 & 35 \\
$\delta$-BHC & 71 & 35 \\
$p, p^{\prime}$-DDE & 35 & 282 \\
$o, p^{\prime}$-DDT & 35 & 71 \\
$p, p^{\prime}$-DDD & 35 & 71 \\
$p, p^{\prime}$-DDT & 35 & 71 \\
\hline
\end{tabular}

Table 2. Recoveries of BHCs and DDTs from Kamishoyosan

\begin{tabular}{|c|c|c|c|c|c|c|c|c|}
\hline \multirow{3}{*}{$\frac{\text { Spiked level (ppm) }}{\text { Compound }}$} & \multicolumn{6}{|c|}{ Intra-day } & \multirow{2}{*}{\multicolumn{2}{|c|}{$\begin{array}{c}\text { Inter-day } \\
0.2\end{array}$}} \\
\hline & \multicolumn{2}{|c|}{0.04} & \multicolumn{2}{|c|}{0.2} & \multicolumn{2}{|c|}{1.0} & & \\
\hline & $\begin{array}{c}\text { Average } \\
(\%)\end{array}$ & $\begin{array}{l}\mathrm{RSD} \\
(\%)\end{array}$ & $\begin{array}{c}\text { Average } \\
(\%)\end{array}$ & $\begin{array}{l}\mathrm{RSD} \\
(\%)\end{array}$ & $\begin{array}{c}\text { Average } \\
(\%)\end{array}$ & $\begin{array}{l}\mathrm{RSD} \\
(\%)\end{array}$ & $\begin{array}{c}\text { Average } \\
(\%)\end{array}$ & $\begin{array}{l}\text { RSD } \\
(\%)\end{array}$ \\
\hline$\alpha-\mathrm{BHC}$ & 83.6 & 2.7 & 91.4 & 1.9 & 103.3 & 3.9 & 93.3 & 1.8 \\
\hline$\gamma-\mathrm{BHC}$ & 82.8 & 7.1 & 91.8 & 1.9 & 101.8 & 3.7 & 92.8 & 1.7 \\
\hline$\beta$-BHC & 82.0 & 6.3 & 91.7 & 0.9 & 102.4 & 3.1 & 95.0 & 3.5 \\
\hline$\delta$-BHC & 84.1 & 5.6 & 92.7 & 0.2 & 101.9 & 3.9 & 94.3 & 3.0 \\
\hline$p, p^{\prime}-\mathrm{DDE}$ & 85.1 & 6.5 & 96.9 & 1.1 & 104.9 & 3.6 & 96.9 & 2.1 \\
\hline$o, p^{\prime}-\mathrm{DDT}$ & 92.0 & 9.3 & 101.1 & 10.2 & 111.4 & 3.2 & 104.2 & 3.8 \\
\hline$p, p^{\prime}-\mathrm{DDD}$ & 96.8 & 3.9 & 105.7 & 3.4 & 109.4 & 4.1 & 105.1 & 2.9 \\
\hline$p, p^{\prime}-\mathrm{DDT}$ & 88.0 & 10.5 & 103.3 & 13.3 & 111.4 & 3.3 & 106.7 & 5.1 \\
\hline
\end{tabular}

Inter-day recovery study was carried out over 3 working days. 
and inter-day recovery study (over 3 working days) were conducted for kamishoyosan. Intra-day recovery rates of BHCs and DDTs when spiking solution was spiked at $0.2,1$, and $5 \mu \mathrm{g}$ were between $82.0 \%$ and $111.4 \%$, and the relative standard deviations (RSDs) were between $0.2 \%$ and $13.3 \%$ (Table 2). Inter-day recovery rates of BHCs and DDTs were between $92.8 \%$ and $106.7 \%$, and for RSD were between $1.7 \%$ and $5.1 \%$ (Table 2), indicating these values seemed satisfactory for residue analysis.

The correlation coefficients of linearity are

Table 3. Linear Ranges, Correlation Coefficients and Quantification Limits of BHCs and DDTs

\begin{tabular}{lccc}
\hline \hline Compound & $\begin{array}{c}\text { Range } \\
(\mathrm{ppb})\end{array}$ & $\begin{array}{c}\text { Correlation } \\
\text { coefficient }(\gamma)\end{array}$ & $\begin{array}{c}\text { quantification } \\
\text { limit }(\mathrm{ppb})\end{array}$ \\
\hline$\alpha$-BHC & $5-500$ & 0.9999 & 1 \\
$\gamma$-BHC & $5-500$ & 0.9999 & 1 \\
$\beta$-BHC & $5-500$ & 0.9999 & 2 \\
$\delta$-BHC & $5-500$ & 1.0000 & 1 \\
$p, p^{\prime}$-DDE & $5-500$ & 0.9998 & 1 \\
$o, p^{\prime}-\mathrm{DDT}$ & $5-500$ & 0.9974 & 3 \\
$p, p^{\prime}-\mathrm{DDD}$ & $5-500$ & 0.9997 & 2 \\
$p, p^{\prime}$-DDT & $5-500$ & 0.9961 & 4 \\
\hline
\end{tabular}

shown in Table 3 and varied from $0.9961-1.0000$, showing good linearity.

It was attempted to apply the proposed method to 8 other kampo formulae for which self-imposed residual pesticide limits are set. The recovery values of BHCs and DDTs were between $71.4 \%$ and $116.7 \%$, and most RSD values were less than $10 \%$ (Table 4). SIM chromatograms of shosaikoto (blank sample) and standard solution $(0.05 \mathrm{ppm})$ are shown in Fig. 1. There were no interfering peaks around the pesticide peaks. There were also no interfer-

Table 4. Recoveries of BHCs and DDTs in 8 Other Kampo Products $(n=3)$

\begin{tabular}{lcc}
\hline \hline $\begin{array}{l}\text { Kampo } \\
\text { formulae }\end{array}$ & $\begin{array}{c}\text { Average } \\
(\%)\end{array}$ & $\begin{array}{c}\text { RSD } \\
(\%)\end{array}$ \\
\hline Hochuekkito & $97.1-111.8$ & $0.9-4.6$ \\
Daikenchuto & $71.4-99.5$ & $3.5-7.0$ \\
Saireito & $92.3-111.6$ & $0.7-17.6$ \\
Shosaikoto & $87.5-104.2$ & $0.1-4.4$ \\
Bakumondoto & $87.6-100.1$ & $0.7-4.0$ \\
Goshajinkigan & $99.9-116.7$ & $1.3-3.3$ \\
Rikkunshito & $91.2-106.4$ & $2.0-11.7$ \\
Shoseiryuto & $88.4-105.7$ & $0.5-3.4$ \\
\hline
\end{tabular}
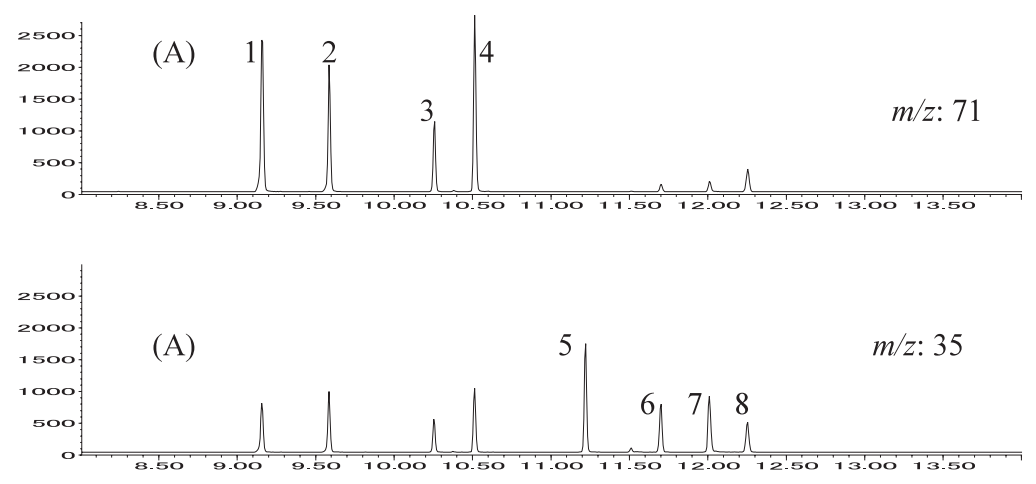

Time (min)

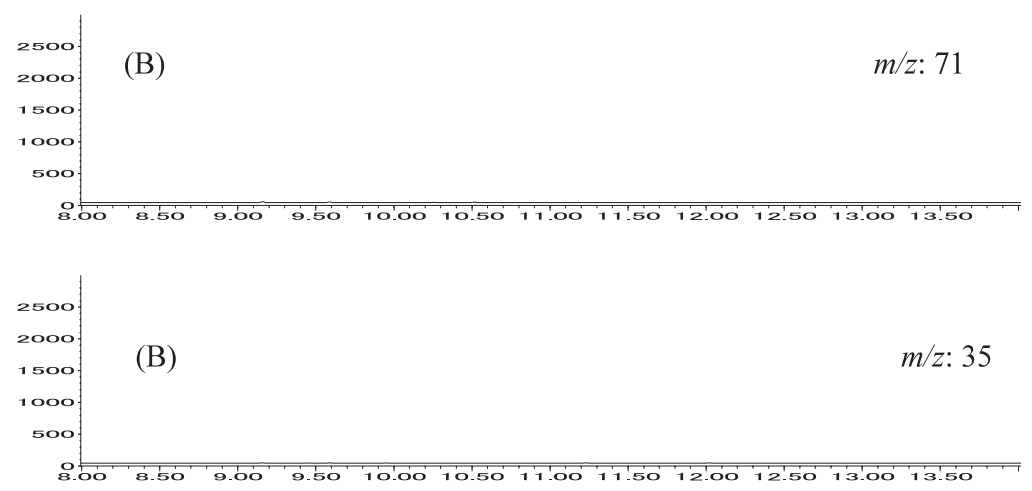

Time (min)

Fig. 1. SIM Chromatograms of (A) Standard Solution (Each Pesticide: $0.05 \mathrm{ppm}$ ), (B) Extract from Shosaikoto (Blank Sample) 1: $\alpha$-BHC, 2: $\gamma$-BHC, 3: $\beta$-BHC, 4: $\delta$-BHC, 5: $p, p^{\prime}$-DDE, 6: $o, p^{\prime}$-DDT, 7: $p, p^{\prime}$-DDD, 8: $p, p^{\prime}$-DDT. 
ing peaks around the pesticide peaks in the sample solutions of other 8 kampo products including kamishoyosan.

Twenty-three samples of kampo products [9 kampo formulae (kamishoyosan, hochuekkito, daikenchuto, saireito, shosaikoto, bakumondoto, goshajinkigan, rikkunshito and shoseiryuto)] were analyzed according to the proposed method. No samples were contaminated by BHCs or DDTs over the quantification limit (as shown in Table 3: concentration in the injected solution; peak height was over 10 times the noise level of the major ions).

In conclusion, a simple, reliable, rapid, and inexpensive sample preparation method was developed to determine BHCs and DDTs in kampo products. Twenty-three samples of kampo products were analyzed according to the proposed method and no samples contained BHCs or DDTs over the quantification limit.

\section{REFERENCES}

1) Shioda, H., Takano, I., Seto, T., Yasuda, I., Hamano, T., Sato, K., Nagayama, Y., Onishi, K., Fujita, S., Ohno, T. and Kajikazawa, T. (1997) Pesticide residues in crude drugs and kampo pharmaceuticals. Annual Report of Tokyo Metropolitan Research Laboratory of Public Health, 48, 67-70.

2) Yoshioka, N., Akiyama, Y., Mitsuhashi, T., Hatanaka, H., Tsuji, M. and Matsushita, S. (2000) Analysis of pesticide residues in Ginseng and Senna. Iyakuhin Kenkyu, 31, 225-231 (in Japanese).

3) Nakazato, M., Nagayama, T., Kan, K., Tabata, S., Tateishi, Y. and Yasuda, I. (2000) Survey of harmful substances in crude drugs (III)_-Ginseng radix-. Annual Report of Tokyo Metropolitan Research Lab- oratory of Public Health, 51, 34-39.

4) Tamura, M., Endo, Y., Isozaki, T., Sugimoto, C., Maruta, J., Ohashi, S., Yoshikawa, S., Okonogi, A., Shimizu, K. and Sasaki, H. (2008) Pesticide residues in kampo formulations, crude drug formulations and crude drugs (1). Iyakuhin Kenkyu, 39, 63-75 (in Japanese).

5) Shioda, H., Hamano, T., Nakajima, J. and Yasuda, I. (2007) Determination of organochlorine and pyrethroid pesticides in preparations of crude drug extract. Annual Report of Tokyo Metropolitan Institute of Public Health, 58, 123-125.

6) Tagami, T., Kajimura, K., Takagi, S., Satsuki, Y., Nakamura, A., Okihashi, M., Akutsu, K., Obana, H. and Kitagawa, M. (2007) Simultaneous analysis of 17 organochlorine pesticides in natural medicines by GC/MS with negative chemical ionization. Yakugaku Zasshi, 127, 1167-1171.

7) Tagami, T., Kajimura, K., Yamasaki, K., Sawabe, Y., Nomura, C., Taguchi, S. and Obana, H. (2009) Simple and rapid determination of cypermethrin and fenvalerate residues in kampo products by gas chromatography/mass spectrometry with negative chemical ionization. J. Health Sci., 55, 777-782.

8) Japan Kampo Medicine Manufacturers Association (2004), Kanpouseizainadonoseisandoutai (in Japanese), http://www.nikkankyo.org/publication/ movement/h16pdf/04_05.pdf.

9) Kajimura, K., Sakagami, Y., Yokoyama, H. and Doi, S. (1994) Analytical method for organochlorine pesticide residues in crude drug containing essential oil. Proceeding of Osaka Prefectural Institute for Public Health ED. of Pharmaceutical Affairs, 28, 63-68.

10) Terasaki, S. and Tsuno, T. (2004) Analytical method for organochlorine pesticide residues in crude drugs. Annual Report of Toyama Prefectural Institute for Pharmaceutical Research, 31, 38-40. 